\title{
Examination of $\mathrm{pH}$, Titratable Acidity and Antioxidant
} \section{Activity in Fermented Milk}

\author{
Julijana Tomovska, Nikola Gjorgievski, Borche Makarijoski \\ Faculty of Biotechnical sciences - Bitola; University, "St. KlimentOhridski”- Bitola, Bitola 7000, R. Macedonia
}

\begin{abstract}
As a fermentation medium homogenized and sterilized whole milk is used. The length of the fermentation was 4 hours, symbiosis of Streptococcus thermophilus and Lactobacillus delbruesciissp. Bulgaricus were used, to achieve the isoelectric point of casein $4.6 \mathrm{pH}$. Monitored parameters were: $\mathrm{pH}$, titratable acidity and antioxidant activity (AOA) in a period of 15 days. It has been determined a decrease of $\mathrm{pH}$ value from 6.67 to 4.19 , an increase of titratable acidity from 8.0 to $36.07{ }^{\circ} \mathrm{SH}$ and an enhancement of AOA from 6.13 to $47.4 \%$. The determination of AOA was accordeda method which is used for different types of food, by using stable 2,2-diphenyl-1-pikrilhidrazil (DPPH) free radical and the absorbance was measured at $517 \mathrm{~nm}$ on a spectrophotometer Spectroquant Pharo 300, Merck. The neutralization activity of the free radical was expressed as a percentage of inhibition of absorbance of DPPH. Milk fermented with Lactobacillus delbrueckii ssp. Bulgaricus, the highest level of AOA registered immediately after the process of fermentation with a value of $52.44 \%$. The lowest value of AOA for the same symbiosis of cultures was observed at the third day after fermentation process kept at a temperature of $4{ }^{\circ} \mathrm{C}$, with a value of $39.43 \%$.
\end{abstract}

Key words: Yogurt, fermented products, $\mathrm{pH}$, titratable acidity, antioxidant activity.

\section{Introduction}

\subsection{Fermented Dairy Products as Functional Foods}

The components of the milk provide important nutritional elements, immune protection and are biologically active substances, and are very important for infants and adult individuals. Lately there are a number of scientific papers [1], which prove and confirm that the consumption of fermented dairy products shows great contributions in maintaining health, which can be considered to be more important than the nutritional value of milk.

Role of $\mathrm{pH}$ monitoring in making yogurt is crucial in producing consistent, quality yogurt. Most yogurts are inoculated with a starter culture consisting of Lactobacillus Bulgaricus and Streptococcus thermophilus. Once the live culture is added, the mixture of milk and bacteria is incubated, allowing for the conversion of lactose to lactic acid. As lactic acid

"Corresponding author: Julijana Tomovska, Ph.D., professor D-r of chemistry, research field: chemistry in food, especially in milk. is produced, there is a corresponding in $\mathrm{pH}$ drop. Most producers have a set point between $\mathrm{pH} 4.0$ and 4.6 in which fermentation is arrested by rapid cooling. The amount of lactic acid present at this $\mathrm{pH}$ level is ideal for a yogurt, giving it the characteristic tartness, aiding in thickening, and acting as a preservative against undesirable strains of bacteria [2].

Relationship between titratable acidity (TA) and $\mathrm{pH}$ during lactic acid fermentation were important. TA and $\mathrm{pH}$ measurements were taken during fermentation lactic acid reconstituted nonfat milk ranging from $9 \%$ to $20 \%$ solids. Measurements were made of dissolved products and also the respective whey derived from them, the ratio of TA, concentration of the $\mathrm{pH}$, and solids during the fermentation to $4.4 \mathrm{pH}$ [3]. The mechanisms of the reactions responsible for the antioxidant characteristics of milk, characteristics attributed to a number of bioactive peptides found naturally in milk or are released into it during the technological process of fermentation [4]. Fermented dairy products have significant impact on human health due to the release of bioactive peptides during 
process of fermentation [5]. The consumption of fermented dairy products reduces the level of cholesterol in blood serum [6]. Fermented dairy products have an antihypertensive effect and protective effect on the skeletal system [7-9].

\subsection{Antioxidant Systems in Milk}

Auto lipid oxidation in milk is a complex network of pro-oxidants and antioxidants. Among the antioxidant enzymes, superoxide dismutase (SOD) is an enzyme that alternately catalyzes the dismutation of the superoxide radical into either ordinary molecular oxygen or hydrogen peroxide superoxide anion of the hydrogen peroxide. Furthermore, the degradation of hydrogen peroxide can be catalyzed by catalase glutathione peroxidase selenium protein. Catalase, also decomposes the lipid peroxide. Antioxidant vitamins in milk provide a large contribution to the daily intake of these dietary components. For example, vitamin E and carotenoids present a fat-soluble antioxidants, which are found in the membranes of the fat globules, which indeed occurs auto oxidation milk fat. Vitamin $\mathrm{C}$ as part of the milk anti-oxidative system is of great importance as part of the conversion radicaltocopherol oxy in tocopherol [10]. For lactoferinn supposed that plays an important role in the binding of pro-oxidative iron ions which prevents fat oxidation and the formation of free peroxy radical, attributed more features including: connecting the iron ions plays a role in the absorption of iron, bacteriostatic or bactericidal activity as and represents growth factor [11]. Concentration of antioxidant systems in milk is in great depending the method of feeding the cows and by the way storing milk. Antioxidant activity determined by DPPH assay showed activity in probiotic fermented milk obtained from all the products being highest in goat milk (93\%) followed by product from camel milk (86\%) and then product from cow milk (79\%) [12]. In milk is present greater number of antioxidant systems, possibly a number of specific reactions for each antioxidant, and the specific function of each antioxidant it can't be defined. There are indications that other components in the milk can show antioxidant function, so that the measurement of the total antioxidant capacity can be a useful method for determining the role of these components [10].

\section{Material and Method}

As a medium for this surveyis used whole homogenised and sterilized cow's milk, that has the following average chemical composition, average chemical composition of the data comes from the declaration of quality given by the manufacturer of the packaging of milk: proteins $3.1 \mathrm{~g}$, milk fat $3.2 \mathrm{~g}$, lactose $4.5 \mathrm{~g}$ and calcium $120 \mathrm{mg} / \mathrm{ml}$. The milk was fermented with different starter cultures as follow: Lactobacillus casei, Lactobacillus acidophilus, Bifidobacterium bifidus, as well as was used the symbiosis of Streptococcus thermophilus and Lactobacilus delbrueckii ssp. Bulgaricus. The milk fermentation process was performed according to the standards of the dairy industry and the instructions for use of starter culture manufacturer CHR Hansen Denmark. Parameters that were monitored and later statistical processed were as follows: Active acidity, $\mathrm{pH}$, titratable acidity in Soxlet-Henkel expressed in ${ }^{\circ} \mathrm{SH}$, antioxidant activity (AOA) expressed in $\%$ of the neutralization of free radical. After the fermentation was completed, the parameters were observed in the first, third, fifth, 10th and 15th day. The methods used for determining the antioxidant activity and the method of preparing samples for analysis was applied [13-15], with some modifications necessary for adapting our surroundings, conditions in the laboratory where the tests were conducted.

\subsection{Preparation of Fermented Milk - Yogurt}

As a fermentation medium whole homogenized and sterilized cow's milk is used. Before fermentation process milk was heated in a sterilized container at temperature of $35^{\circ} \mathrm{C}$. Inoculation was done by direct 
seeding of culture in the required amount of milk with concentration of $0.01 \% \mathrm{w} / \mathrm{v}$, and that represents the industry standard for fermented products manufacture. After seeding the contents were mixed 5 to 10 minutes with sterile blender for achieving better dispersion of the culture in the medium. Before use the edge of the bag where the cultures were sterilized with ethanol. Also the equipment for cutting and weighing container was sterilized with ethanol. Fermentation was performed in a sterile disposable container and thermal chamber at temperature of $40{ }^{\circ} \mathrm{C}$. The length of the fermentation was 4 hours using starter culture that was symbiosis of streptococcus thermophilus and Lactobacillus delbruescii ssp. Bulgaricus, and 12 hours at samples fermented by Lactobacillus casei, L. acidophilus and Bifidobacterium bifidus. Fermentation is completed when case in reaches its isoelectric point of $4.6 \mathrm{pH}$. When the process of fermentation was finished, all the samples were taken to the refrigerator for storage at a temperature of $4{ }^{\circ} \mathrm{C}$, up to the next control measurement of the examined samples.

\subsection{Measurement of $\mathrm{PH}$}

The active acidity or $\mathrm{pH}$ is measured in raw milk prior to fermentation and immediately after completion of the fermentation with the appropriate culture. Also the $\mathrm{pH}$ was measured at each control point. For $\mathrm{pH}$ measurement, digital $\mathrm{pH}$ meter LAB-860 was used.

\subsection{Measurement of Titratable Acidy}

The measurement of titratable acidity was according the method used that is described in Ref. [16]. Erlenmeyer flask used with a transfer pipette $20 \mathrm{ml}$ milk or yogurt and $1 \mathrm{ml}$ of $2 \% \mathrm{w} / \mathrm{v}$ solution of phenolphthalein. Content is titrated with $0.1 \mathrm{M} \mathrm{NaOH}$ solution to appearance the faint pink color that will not get lost for over 2 minutes. Acidification of milk (yogurt) is calculated by the formula: $\mathrm{K}=\mathrm{V} \cdot 2$, where $\mathrm{V}$ - volume is consumed base neutralization.

\subsection{Antioxidant Activity - Preparation of the Sample for Analysis}

Whey fraction was prepared as described by [15], and was used immediately after preparation, have some changes that were necessary to adapt the method to the working conditions and equipment in the laboratory. Not hydrolyzed casein was removed. Doses of $15 \mathrm{ml}$ are taken from each sample and the $\mathrm{pH}$ was adjusted to 4.6 (only for samples with higher $\mathrm{pH}$ of 4.6 and with unfermented milk) with $1 \mathrm{M} \mathrm{HCl}$. The suspension was centrifuged in centrifuge at 9,000 $\mathrm{rpm}^{-1}, \mathrm{RCF}=7,690 \mathrm{~g}$ for 20 minutes in a centrifuge Hettich Universal $320 \mathrm{R}$ (Andreas Hettich GmbH-Germany), and the supernatant was filtered through coarse filter paper.

\subsection{Determination of the Ability to Neutralize Free} Radical

This simple method is developed for determination of antioxidant activity of various foods and uses the 2,2-diphenyl-1-pikrilhidrazil (DPPH) free radical. The structure of DPPH and its reduction are shown in Fig. 1. No paired electron of DPPH gives the strong absorption of $517 \mathrm{~nm}$ and has a purple color. The color changes from purple to yellow as the molar absorption at this wavelength radical decreases when no paired electron gets another electron and it is paired. This electron donor is a neutralizer of free radicals of the test medium. The resulting bleaching stoichiometry is the number of trapped electrons [17].

The neutralizing activity of DPPH method is determined in Ref. [13]. DPPH was used as a stable radical. Volume of $2 \mathrm{ml}$ of DPPH in ethanol solution (500 $\mathrm{mM}$ ) was added to $2 \mathrm{ml}$ of whey faction, the sample is intensively mixed and allowed to stand at room temperature in a dark place for 30 minutes. Absorbance was measured at $517 \mathrm{~nm}$ wavelength Spectroquant Pharo 300 spectrophotometer from manufacturer Merck. Ethanol was used as a calibration solution as a reagent was used DPPH solution in ethanol. The neutralization activity of the 

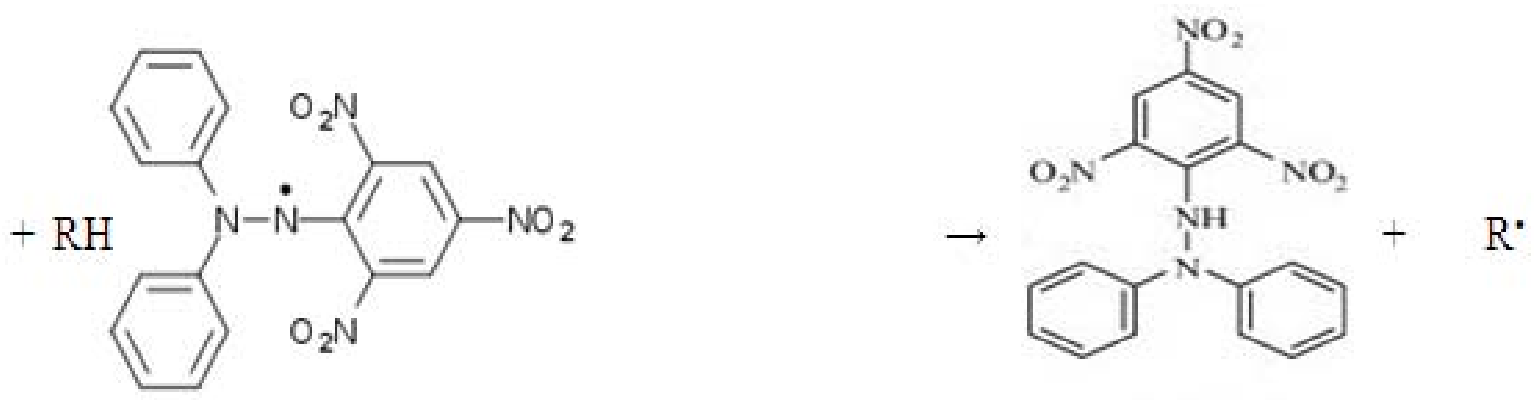

DPPH-H

Fig. 1 Structure of DPPH radical and a reduction.

Table 1 Determination of $\mathrm{pH}$ in milk samples fermented with different cultures.

\begin{tabular}{lllll}
\hline Days & L. bulgaricus + S. thermophillus & L. Casei & L. Acidophillus & B. Bufidus \\
\hline $0^{* * *}$ & 6.67 & 6.67 & 6.67 & 6.67 \\
0 & 4.47 & 4.46 & 4.2 & 4.48 \\
1 & 4.4 & 4.34 & 4.13 & 4.39 \\
3 & 4.31 & 4.29 & 4.1 & 4.37 \\
5 & 4.32 & 4.28 & 4.14 & 4.49 \\
10 & 4.19 & 4.22 & 4.11 & 4.27 \\
15 & 4.19 & 4.17 & 4.08 & 4.25 \\
\hline
\end{tabular}

**** the value of the fermented milk.

free radical expressed as a percentage of inhibition of the DPPH absorbency according to the following expression:

Inhibition $=[(A$ control $-A$ test $) / A$ control $] \times 100$

where, $A$ control is the absorbance of the blank (DPPH solution in ethanol without whey) and $A$ test is the absorbance of the sample (DPPH with a sample of whey).

\section{Results and Discussion}

3.1 Determination of PH in Milk Fermented with Different Cultures

The aim of this study is following acidity activity and titratable acidity of fermented milk as factors that have impact and changed the antioxidant activity in whole cow's milk. From that point followed the dynamics of change in these two parameters in 15 days, the change of $\mathrm{pH}$ and ${ }^{\circ} \mathrm{SH}$ values from technology aspects.

Parameters examined in sterilized whole milk, which representing a point of comparison and monitoring of the dynamics of fermentation with different types of microbial cultures, have values 6.67 $\mathrm{pH}$ - active acidity of milk, $8{ }^{\circ} \mathrm{SH}$ - titration acidity and $6.13 \%$ AOA - antioxidant activity. The comparison of $\mathrm{pH}$ values of fermented milk samples with different starter cultures is presented in Table 1.

The dynamics of $\mathrm{pH}$ changes in all control points can best be seen from Graph 1. If we compare the values of $\mathrm{pH}$, a decrease was noticed, and that trend continued till the final control point at the 15th day.

3.2 Determination of ${ }^{\circ} \mathrm{SH}$ Values in Fermented Milk Samples with Different Cultures

The values of titratable acidity, expressed in ${ }^{\circ} \mathrm{SH}$ in fermented milk samples with different cultures are presented in Table 2.

The dynamics of titratable acidity ( $\left.{ }^{\circ} \mathrm{SH}\right)$ changes of a different microbial cultures duringthe process of fermentation have been shown in Graph 2.

From Graph 2 it can be seen that the values of titratable acidity are in the normal range of all unfermented milk samples. A significant increase in 


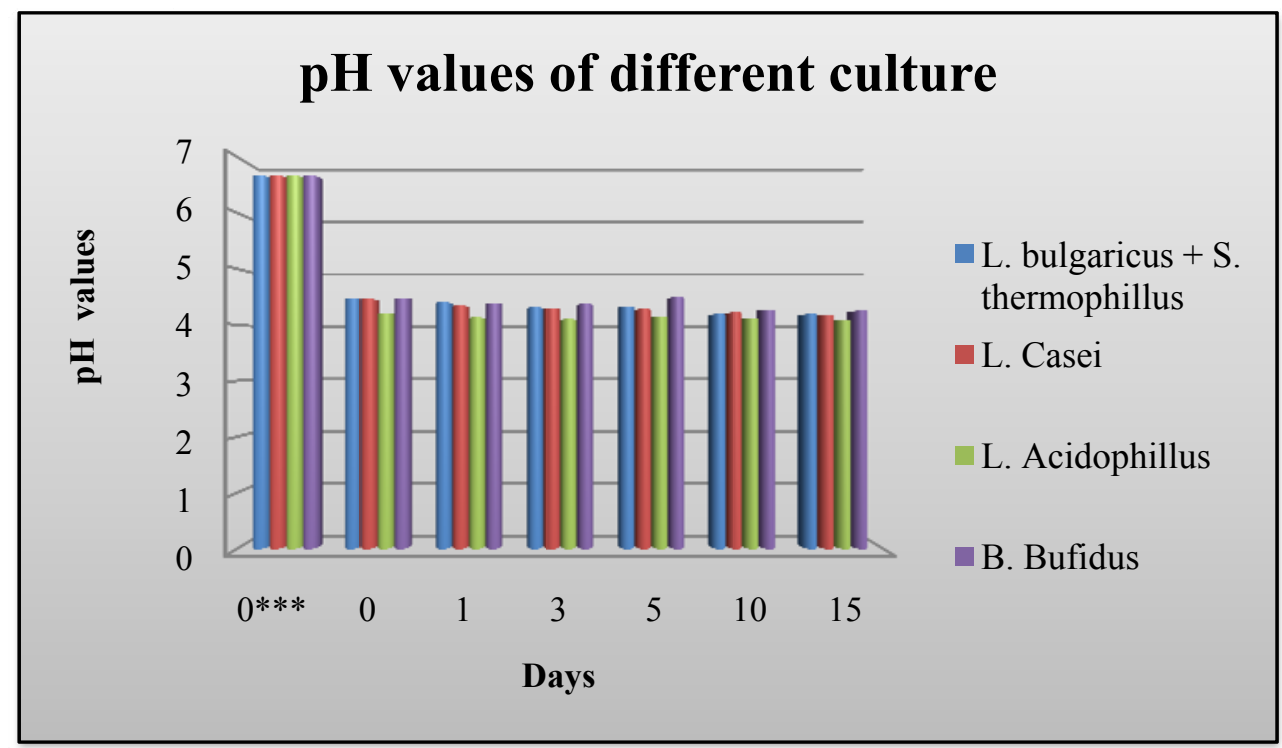

Graph 1 Values of pH fermentation with different culture.

Table 2 Determination of ${ }^{\circ} \mathrm{SH}$ in fermented milk samples with different cultures.

\begin{tabular}{lllll}
\hline Days & L. bulgaricus + S. thermophillus & L. Casei & L. Acidophillus & B. Bufidus \\
\hline $0^{* * *}$ & 8 & 8 & 8 & 8 \\
0 & 27.87 & 28.53 & 34.8 & 32.06 \\
1 & 28.23 & 32.5 & 37.3 & 31.96 \\
3 & 31.07 & 32.46 & 38.2 & 33.33 \\
5 & 31.47 & 33.73 & 37.9 & 33.46 \\
10 & 33.73 & 36.53 & 40.06 & 36.73 \\
15 & 36.07 & 35.86 & 40.06 & 35.9 \\
\hline
\end{tabular}

**** The value of the nonfermented milk.

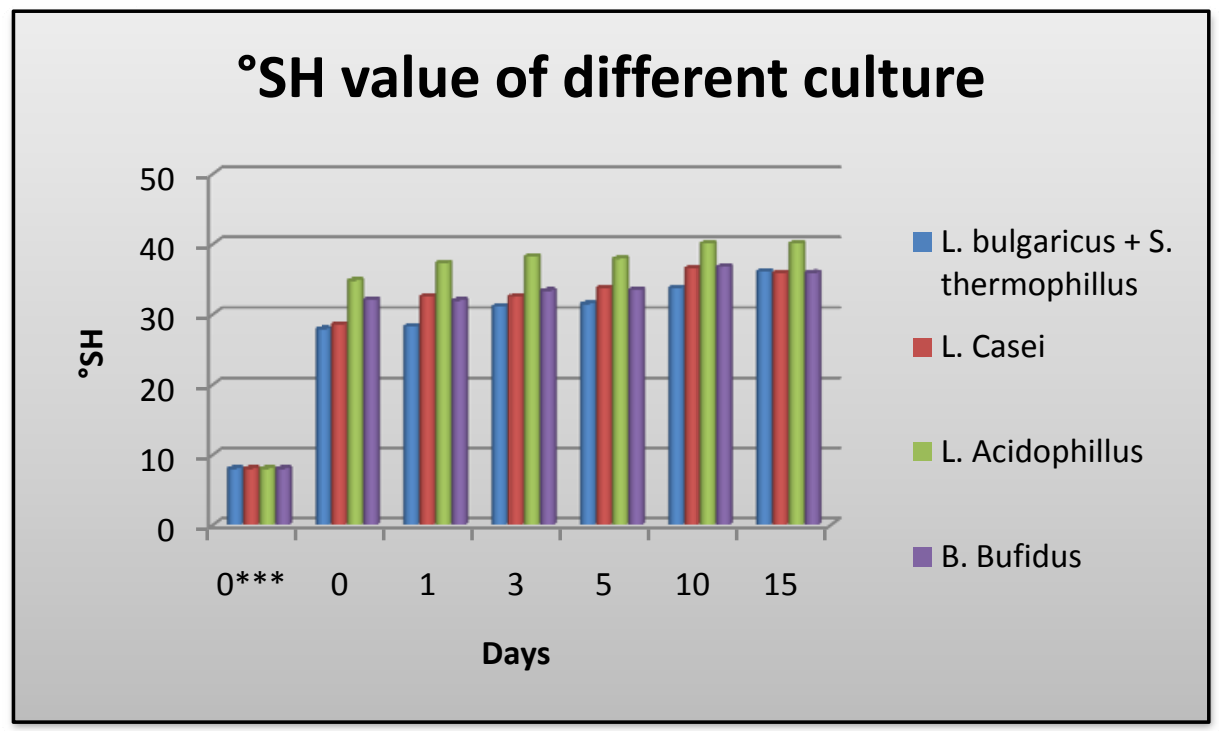

Graph 2 Dynamics of ${ }^{\circ} \mathrm{SH}$ at fermented milk samples with different culture. 
titratable acidity was noticed at the end of the fermentation and an upward trend was kept until the $15^{\text {th }}$ day. According to this data can be concluded that there is an intense protolithic activity in all types of starter cultures, which is closely related to other tested parameters such as antioxidant activity. Also from Fig. 2 can be done mutual comparison of the values obtained in the fermentation using various types starter cultures for all days. Also, it can be mentioned that no significant changes in titratable acidity in the same day were noticed for different types of culture.

3.3 Determination of AOA - Antioxidant Activity

The Antioxidant activity of different microbiological culture in fermented milk are presented in Table 3.

It has been made comparison between AOA of the above mentioned microbial cultures in non-fermented milk samples and significant increase was determined.

In the Graph 3 is presented the dynamic of antioxidant activity (AOA) of applied microbiology cultures during the fermentation process.

Overall, the highest level of AOA was noticedat fermented milk samples with Lactobacillus acidophilus (58.76\%) and same day the lowest level of AOA was noticed at fermented milk samples with symbiosis of streptococcus thermophilus and Lactobacillus delbrueckii ssp. Bulgaricus (45.43\%). The highest level of AOA of milk samples fermented

Table 3 Values for antioxidant activity of assayed microbiological cultures.

\begin{tabular}{lllll}
\hline Days & L. bulgaricus + S. thermophillus & L. Casei & L. Acidophillus & B. Bufidus \\
\hline $0^{* * *}$ & 6.13 & 6.13 & 6.13 & 6.13 \\
0 & 52.44 & 56.51333 & 63.99 & 54.93 \\
1 & 45.43 & 49.39 & 58.76667 & 52.13333 \\
3 & 39.43 & 49.06 & 55.67333 & 46.46 \\
5 & 43.01 & 47.60667 & 49.48333 & 44.03 \\
10 & 43.34 & 52.87 & 48.60333 & 42.26333 \\
15 & 47.42 & 54.55333 & 52.63667 & 45.31333 \\
$\bar{X} *$ & 45.17833 & 51.66556 & 54.85889 & 47.52167 \\
SD** & 4.448476 & 3.51376 & 5.869891 & 4.939566 \\
\hline
\end{tabular}

*Average value without baseline for unfermented milk.

**Standard deviation without baseline for unfermented milk.

*** Baseline unfermented milk.

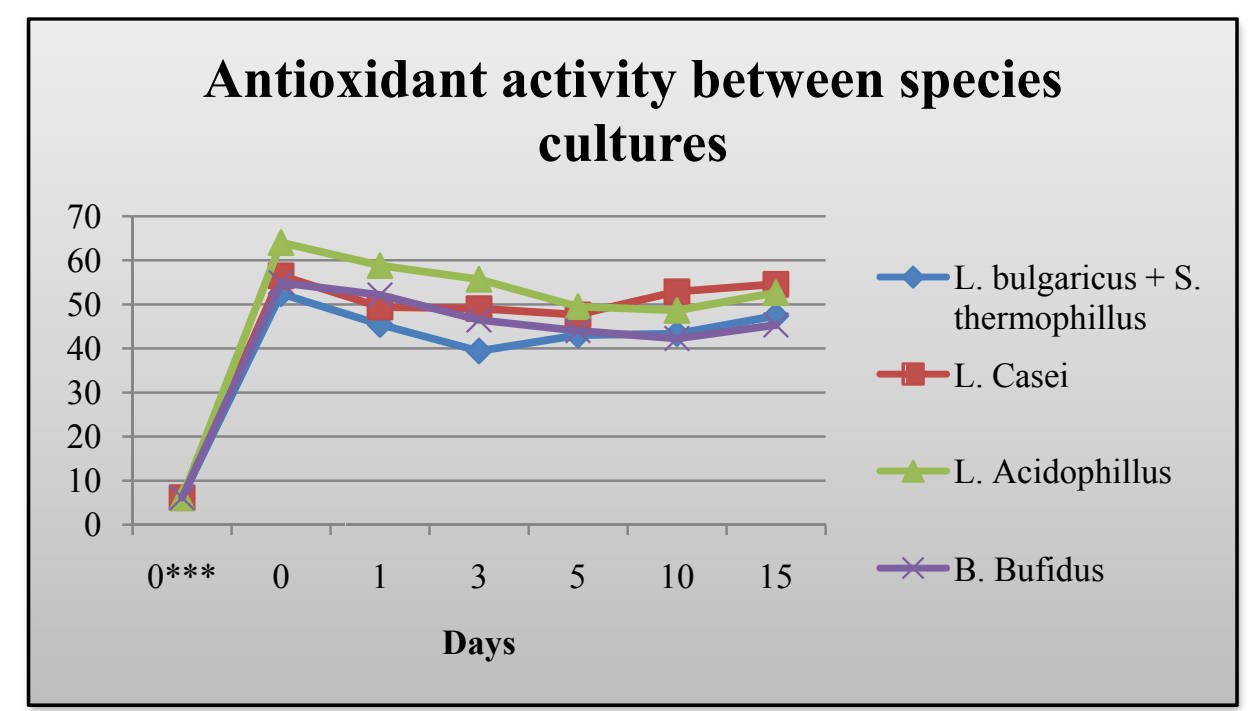

Graph 3 Values obtained for antioxidant activity. 
with Lactobacillus delbrueckii ssp. Bulgaricus, was registered at 0 s day or immediately after fermentation with a value of $52.44 \%$. The lowest value of AOA for the same symbiosis of cultures was noticed at the third day of fermentation kept at a temperature of $4{ }^{\circ} \mathrm{C}$, with a value of $39.43 \%$. The highest level of AOA of milk samples fermented with Lactobacillus acidophilus was registered also after completion of fermentation process and its value was $63.99 \%$, and the lowest value for AOA was noticed at the $10^{\text {th }}$ day of storage at a temperature of $4{ }^{\circ} \mathrm{C}$, measured with $48.60 \%$, neutralized free radicals. Milk samples fermented by Lactobacillus casei, also noticed the highest level of $56.51 \%$ after finishing the fermentation process and the lowest value of AOA on the fifth day after fermentation kept at $4{ }^{\circ} \mathrm{C}$ with $47.61 \%$ neutralization of DPPH free radical. The highest level of AOA of milk samples fermented with Bifidobacterium bifidus was also noticed after a fermentation process, with a value of $54.93 \%$. The lowest value of AOA was noticed at the 10th day of storage at a temperature of $4{ }^{\circ} \mathrm{C}$, measured $42.26 \%$ neutralization of DPPH free radical. The difference of the values for the antioxidant activity may also be dependent on the conditions in the laboratory during the examinations, such as the conditions under which the fermentation takes place, sensitivity of instruments used and the sanitary conditions in the laboratory [18].

\section{Conclusions}

According to the analyzed data, we can conclude the following points:

During the fermentation process a great increase in titratable acidity from 8 until $40.0^{\circ} \mathrm{SH}$ had in all samples of milk most with Streptococcus thermophilus and Lactobacillus delbrueckii ssp. Bulgaricus, was observed.

Fermented milk samples obtained from different microbial cultures have shown a higher value in titratable acidity and antioxidant activity, compared with the values of the same parameters in unfermented milk samples.

The high antioxidant activity was registered at milk fermented with Lactobacillus acidophilus.

The highest level of AOA was measured immediately after the end of fermentation and its value was $63.99 \%$.

The lowest level of AOA was measured in milk fermented with the symbiosis of Streptococcus thermophilus and Lactobacillus delbrueckii ssp. Bulgaricus, on the third day-39.43\%.

\section{References}

[1] Parra, M. D., Martinez de Morentin, B. E., Cobo, J. M., Mateos, A. and Martinez, J. A. 2004. "Daily Ingestion of Fermented Milk Containing Lactobacillus Casei DN114001 Improves Innate-Defense Capacity in Healthy Middle-Aged People." J Physiol Biochem 60: 85-91.

[2] Masulli, D. 2016. "Measuring PH of Yogurt." Food Quality \& Safety July 15.

[3] Wilkowske, H. H. 1956. "Relationship between Titratable Acidity and PH during Lactic Acid Fermentation in Reconstituted Nonfat Milk." Journal of Dairy Science 174, 37 (1): 22-9.

[4] Shah, N. 2007. "Functional Cultures and Health Benefits." Int. dairy J. 17: 1262-77.

[5] Jäkälä, P. and Vapaatalo, H. 2010. "Antihypertensive Peptides from Milk Proteins." Pharmaceuticals 3: 251-72.

[6] Kawase, M., Hashimoto, H., Hosoda, M., Morita, H. and Hosono, A. 2000. "Effect of Administration of Fermented Milk Containing Whey Protein Concentrate to Rats and Healthy Men on Serum Lipids and Blood Pressure." J Dairy Sci. 83: 255-63.

[7] Seppo, L., Jauhiainen, T., Poussa, T. and Korpela, R. 2003. "A Fermented Milk High in Bioactive Peptides has a Blood Pressure-Lowering Effect in Hypertensive Subjects." Am J Clin Nutr 77: 326-30.

[8] Tomovska, J., Presilski, S., Gjorgievski, N., Tomovska, N., Qureshi, M. N. and Bozinovska, N. P. 2013. "Development of a Spectrophotometric Method for Monitoring Angiotensin-Converting Enzyme in Dairy Products." Pak. Vet. J. 33 (1): 14-8.

[9] Moller, N. P., Scholz-Ahrens, K. E., Roos, N. and Schrezenmeir, J. 2008. "Bioactive Peptides and Proteins from Foods: Indication for Health Effects." Eur J Nutr 47: 171-82.

[10] Lindmark-Månsson, H. and Akesson, B. 2000. “Antioxidative Factors in Milk.” Br J Nutr. 84 (Suppl 1): 
S103-10.

[11] Bihel, S. and Birlouez-Aragon, I. 1998. "Inhibition of Tryptophan Oxidation in the Presence of Iron-Vitamin C by Bovine Lactoferrin." International Dairy Journal 8: 637- 41.

[12] Balakrishnan, G. and Agrawal, R. 2014. "Antioxidant Activity and Fatty Acid Profile of Fermented Milk Prepared by Pediococcus." J Food Sci. Technology 51 (12): 4138-42.

[13] Son, S. and Lewis, B. A. 2002. "Free Radical Scavenging and Antioxidative Activity of Caffeic Acid Amide and Ester Analogues: Structure- Activity Relationship." J. Agric. Food Chem. 50: 468-72.

[14] Ou, C. C., Lu, T. M., Tsai, J. J., Yen, J. H., Chen, H. W. and Lin, M. Y. 2009. "Antioxidative Effect of Lactic Acid Bacteria: Intact Cells vs. Intracellular Extracts.” J.
Food Drug Anal. 17: 209-16.

[15] Virtanen, T., Pihlanto, A., Akkanen, S. and Korhonen, H. 2007. "Development of Antioxidant Activity in Milk Whey during Fermentation with Lactic Acid Bacteria." J. Appl. Microbial. 102: 106-15.

[16] Caric, M., Milanovic, S. and Vucelja, D. 2000. "Standard Methods for Milk and Milk Products Analysis." Novi Sad, Srbija.

[17] Miller, H. E., Rigelhof, F., Marquart, L., Prakash, A. and Kanter, M. 2000. "Antioxidant Activity. A Publication of Medallion Laboratories." J. Am. Coll. Nutr. 19 (3): 312S-319S.

[18] Gjorgievski, N., Tomovska, J., Dimitrovska, G., Makarijoski, B. and Shariati, M. A. 2014. "Determination of the Antioxidant Activity in Jogurt." Hygienic Engineering and Design (HEDG) 8: 88-92. 